\title{
Mathematical Model and Algorithm for Determination of Minimum Antenna Mast Height for Terrestrial Line of Sight Microwave Link with Zero Path Inclination
}

\author{
Wali Samuel $^{1}$, Steve Worgu ${ }^{2}$, Elsie Chidinma Anderson ${ }^{2}$ \\ ${ }^{1}$ Department of Electrical/Electronic and Computer Engineering, University of Uyo, Uyo, Nigeria \\ ${ }^{2}$ Department of Electrical/ Computer Engineering, Port Harcourt Polytechnic, Rumuola, Port Harcourt, Nigeria \\ Email address: \\ samwalliuy@yahoo.com (W. Samuel), stevewa438@yahoo.com (S. Worgu)
}

\section{To cite this article:}

Wali Samuel, Steve Worgu, Elsie Chidinma Anderson. Mathematical Model and Algorithm for Determination of Minimum Antenna Mast Height for Terrestrial Line of Sight Microwave Link with Zero Path Inclination. International Journal of Systems Science and Applied Mathematics. Vol. 2, No. 1, 2017, pp. 36-41. doi: 10.11648/j.ijssam.20170201.15

Received: October 31, 2016; Accepted: January 4, 2017; Published: January 31, 2017

\begin{abstract}
In this paper, mathematical model and algorithm for determination of minimum antenna mast height for terrestrial line of sight microwave link with zero path inclination is presented. The mathematical expressions developed are used for computing relevant link parameters while the algorithm gives the procedure for using the mathematical expressions for computing the minimum antenna mast heights. Sample $40 \mathrm{~km} 10 \mathrm{GHz} \mathrm{Ku}$-band microwave link is used to demonstrate the application of the model and algorithm; in this case, the transmitter is located at longitude 7.711747 and latitude 5.178536 and the receiver is located at longitude 8.039903 and latitude 5.055223 . The link is required to make a minimum of $100 \%$ clearance with respect to the Fresnel zone 1. The results show that the transmitter and receiver antenna are at the same line of sight height of $158.7 \mathrm{~m}$ whereas the transmitter antenna mast height is $68.8 \mathrm{~m}$ while the receiver antenna mast height is $109.7 \mathrm{~m}$. Also, the maximum obstruction height of $128.58 \mathrm{~m}$ occurred at a distance of $14306.98 \mathrm{~m}$ from the transmitter with percentage clearance of $100 \%$ in respect of Fresnel zone 1 . The result shows that the model can be used to ensure that the specified percentage clearance is achieved through the application of the models presented in this paper.
\end{abstract}

Keywords: Antenna Mast Height, Path Inclination, Microwave Link, Line Of Sight Communication, Percentage Clearance

\section{Introduction}

Microwave signals travel in a straight line $[1,2]$. Consequently, they are used for Line Of Site (LOS) communication. Researches however, have found that microwave signal do not travel in a pencil tip straight line. Rather, the signal spread from the transmitter and terminate at the receiver forming an ellipsoidal shape that is described by Fresnel and modeled as Fresnel zones [3, 4, 5, 6]. Also, secondary radioclimatic parameter called effective earth radius factor (k-factor) affect the signal path [8-10]. At different values of $\mathrm{k}$-factor the signal can bend away from the earth, towards the earth or it can travel in a straight line with respect to the earth surface. Particularly, the effective earth radius factor is dependent on the three primary radioclimatic parameters, namely, temperature, pressure and relative humidity. The effect of the effective earth radius factor is modeled using the concept of earth bulge [11].
Importantly, in LOS communication system, the transmitter antenna and the receiver antenna are installed at a height that will ensure that no obstacle obstruct the LOS between the (straight line joining) transmitter and the receiver. In order to ensure that the required LOS clearance is achieved, during the design of the LOS communication link the Fresnel Zone clearance and the earth bulge are taken into consideration along with the elevation profile of the terrain. Also, the path inclination, which is the angle subtended by the LOS with the horizontal is considered in the determination of the antenna mast height. In the case where the path inclination is zero, it means that the transmitter and the receiver antenna heights are equal. However, that does not mean that the transmitter mast and the receiver antenna mast heights are equal.

In any case, there are several approaches that can be used to determine the minimum transmitter mast and the receiver 
antenna mast heights when the path inclination is zero. In this paper, one of such methods is presented based on a given Fresnel geometry for LOS link. The method assumes that the required LOS percentage clearance with respect to Fresnel zone 1 is specified along with the expected obstruction height. Also, the path elevation profile is required as input data for the model. The requisite mathematical expressions and algorithm for the computation of the minimum transmitter mast and the receiver antenna mast heights are presented.

\section{Theoretical Background}

\subsection{Fresnel Geometry for the Line of Sight Link}

The Fresnel geometry for the line of sight link is given in figure 1 . In the model, it is assumed that the transmitter has the lower antenna height, especially, when the two antenna heights are not equal. However, if the transmitter antenna height is higher than the receiver antenna height, then the analysis still holds by swapping the receiver subscripts with that of the transmitter.

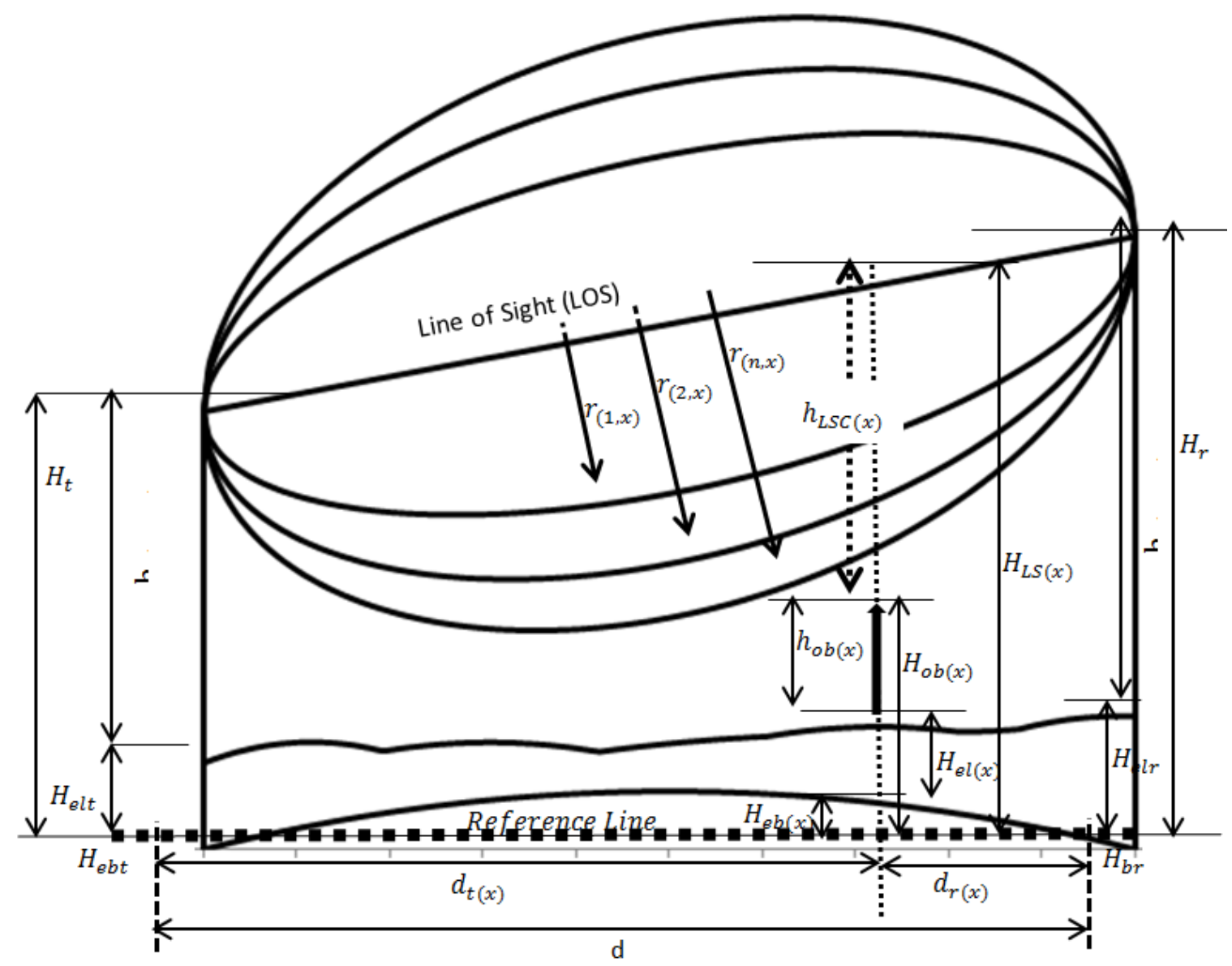

Figure 1. Fresnel Geometry For The Line Of Sight Link.

\subsection{The Elevation Profile}

The model make use of the link elevation profile which is the set of data on the elevation at various points between the transmitter and the receiver. The terrain elevation profile provides a number of elevation points and their distance from the transmitter and the receiver. Each elevation point consist of the elevation height and the distance of the point from the transmitter or distance from the receiver. Let $n_{e}$ be the number of elevation points taken from the transmitter location to the receiver location. Also, let $H_{e l(x)}$ be the elevation at point $\mathrm{x}$, where $\mathrm{x}=1,2,3, \ldots, n_{e}$; let $d_{t(x)}$ be the distance of location $\mathrm{x}$ from the transmitter; let $d_{r(x)}$ be the distance of location $\mathrm{x}$ from the receiver, where $\mathrm{x}=1,2,3, \ldots, n_{e}$ and let $d$ be the distance (in meters) between the transmitter and the receiver. Then,

$$
\begin{aligned}
& d=d_{r(x)}+d_{t(x)} \\
& d_{r(x)}=d-d_{t(x)}
\end{aligned}
$$

Since the transmitter is taken as the reference point for the measurements, then, the transmitter is located at $x=0$ and the receiver is located at $\mathrm{x}=n_{e}$. Therefore,

$$
\begin{aligned}
& d_{t}=d_{t(0)}=0 \\
& d_{r}=d_{t\left(n_{e}\right)}=\mathrm{d}
\end{aligned}
$$

$H_{e l t}$ is the elevation at the transmitter location where $\mathrm{x}=0$, hence, $H_{\text {elt }}=H_{\text {el }(0)}$

$H_{e l r}$ is the elevation at the receiver location where $\mathrm{x}=n_{e}$, hence, $H_{e l r}=H_{e l\left(n_{e}\right)}$ 


\subsection{The Earth Bulge}

Earth bulge is the height an obstruction is raised higher in elevation (into the path) owing to earth curvature. Earth bulge is given as [12];

$$
H_{e b(x)}=\frac{\left(d_{t(x)}\right)\left(d_{r(x)}\right)}{12.75 * K}
$$

Where $H_{e b(x)}$ is the height (in meters) of the earth bulge at location $\mathrm{x}$ between the transmitter and the receiver; $H_{e b t}$ is the height (in meters) of the earth bulge at the transmitter mast location; $H_{e b r}$ is the height (in meters) of the earth bulge at the receiver mast location; $d_{t(x)}$ and $d_{r(x)}$ are as defined earlier. For Line of Sight (LOS) point-to-point links design $\mathrm{K}$-factor of $4 / 3$ is often used. At the transmitter, $d_{t(x)}=0$, hence,

$$
H_{e b t}=H_{e b(x)}=\frac{(0)\left(d_{r(x)}\right)}{12.75 * K}=0
$$

Similarly, at the receiver, $d_{r(x)}=0$, hence,

$$
H_{e b t}=H_{e b(x)}=\frac{\left(d_{t(x)}\right)(0)}{12.75 * K}=0
$$

In essence, at the transmitter and at the receiver, the earth bulge is zero.

\subsection{Radius of the Fresnel Zones}

In many cases obstructions do exist in the signal path. For acceptable clear line of sight, at least $60 \%$ clearance is required in the first Fresnel zone. So, obstructions in the signal path must maintain a clearance height that is at least $60 \%$ of the radius of the first Fresnel none. Theoretically, there are infinite number of Fresnel zones in any LOS link. Let $\lambda$ be the wavelength of the radio wave; let $\mathrm{c}$ be the speed of the radio wave (where $\mathrm{c}=3 \times 10^{8} \mathrm{~m} / \mathrm{s}$ and let $\mathrm{f}$ be the frequency of the radio wave in $\mathrm{Hz}$, then, the radius of the nth Fresnel zone $\left(\mathbf{r}_{(\boldsymbol{n}, \boldsymbol{x})}\right)$ at location $\mathrm{x}$ is given as ]13-15];

$$
\begin{aligned}
& \mathbf{r}_{(\boldsymbol{n}, \boldsymbol{x})}=\sqrt{\frac{n\left(\Lambda\left(d_{t(x)}\right)\left(d_{r(x)}\right)\right)}{\left(d_{t(x)}+d_{r(x)}\right)}} \text {; for } \mathrm{n}=1,2,3, \ldots \text { and } d_{t(x)}>> \\
& \mathbf{r}_{(\boldsymbol{n}, \boldsymbol{x})} \text { and } d_{r(x)}>>\mathbf{r}_{(\boldsymbol{n}, \boldsymbol{x})}
\end{aligned}
$$

$\lambda$ in metres is given as;

$$
\Lambda=\frac{c}{f}
$$

Let $\mathbf{P}_{\boldsymbol{C}(\boldsymbol{n})}$ be the percentage clearance allowed for the Fresnel zone $n$, given in $\%$ where $\mathbf{P}_{\boldsymbol{C}(\boldsymbol{n})}$ is positive if the obstacle tip is below the line of sight and $\mathbf{P}_{\boldsymbol{C}(\boldsymbol{n})}$ is negative if the obstacle tip is above the line of sight. It must be noted that $\mathbf{P}_{\boldsymbol{C}(\boldsymbol{n})}=\mathbf{0} \%$ when the tip of the obstruction is on the LOS; $\mathbf{P}_{\boldsymbol{C}(\boldsymbol{n})}=\mathbf{1 0 0} \%$ when the tip of the obstruction is below the LOS at a clearance height equal to $\mathbf{r}_{\boldsymbol{C}(\boldsymbol{n}, \boldsymbol{x})} ; \mathbf{P}_{\boldsymbol{C}(\boldsymbol{n})}=\mathbf{- 1 0 0} \%$ when tip of the obstruction is above the LOS at a clearance height equal to $\mathbf{r}_{\boldsymbol{C}(\boldsymbol{n}, \boldsymbol{x})}$ above the LOS. Let $\mathbf{h}_{\boldsymbol{C l}(\boldsymbol{n}, \boldsymbol{x})}$ be the clearance height required at point $\mathrm{x}$ for clear line of sight with respect to the Fresnel zone n. Then,

$$
\mathbf{h}_{\boldsymbol{C l}(\boldsymbol{n}, \boldsymbol{x})}=\left(\frac{\mathbf{P}_{\boldsymbol{C}(\boldsymbol{n})}}{\mathbf{1 0 0}}\right) \mathbf{r}_{(\boldsymbol{n}, \boldsymbol{x})}=\left(\frac{\mathbf{P}_{\boldsymbol{C}(\boldsymbol{n})}}{\mathbf{1 0 0}}\right) \sqrt{\frac{n\left(\kappa\left(d_{t(x)}\right)\left(d_{r(x)}\right)\right)}{\left(d_{t(x)}+d_{r(x)}\right)}}
$$

\subsection{Obstruction Location and Height}

In the model, the reference line for measuring the earth bulge is the chord (line segment) that joins the sea level at the transmitter and the sea level at the receiver. In the model of figure 1 the Reference Line is represented by thick dotted horizontal line at the base of figure 1 . Let $\mathrm{h}_{\mathrm{ob}(\mathrm{x})}$ be the height of the obstruction at point $\mathrm{x}$, where $\mathrm{h}_{\mathrm{ob}(\mathrm{x})}$ is measured from the ground level (where the ground level is at the top of the elevation point at point $\mathrm{x}$ ) and it does not include the elevation and earth bulge at point $\mathrm{x}$. The elevation point is measured from the sea level. Let $\mathrm{H}_{\mathrm{ob}(\mathrm{x})}$ be the overall height of the obstruction at point $\mathrm{x}$, where $\mathrm{H}_{\mathrm{ob}(\mathrm{x})}$ is measured from the Reference Line. $\mathrm{H}_{\mathrm{ob}(\mathrm{x})}$ includes the elevation at point $\mathrm{x}$ and also include the earth budge at point $\mathrm{x}$, Then;

$$
H_{o b(x)}=h_{o b(x)}+H_{e b(x)}+H_{e l(x)}
$$

\subsection{Determination of Minimum LOS Height at Location $x$}

For the specified percentage clearance $\mathbf{P}_{\boldsymbol{C}(\boldsymbol{n})}$, the minimum LOS height at location $\mathrm{x}$ is given as $\mathrm{H}_{\mathrm{LSR}(\mathrm{x})}$, where;

$$
\begin{gathered}
\mathbf{H}_{\mathbf{L S R}(\mathbf{x})} \geq H_{o b(x)}+\mathbf{h}_{C l(\boldsymbol{n}, \boldsymbol{x})} \\
\mathrm{H}_{\mathrm{LSR}(\mathrm{x})} \geq H_{e b(x)}+H_{e l(x)}+h_{o b(x)}+ \\
\left\{\left(\frac{\mathbf{P}_{C(\boldsymbol{n})}}{\mathbf{1 0 0}}\right) \sqrt{\frac{n\left(\Lambda\left(d_{t(x)}\right)\left(d_{r(x)}\right)\right)}{\left(d_{t(x)}+d_{r(x)}\right)}}\right\}
\end{gathered}
$$

Let $X_{L S M A X}$ be the distance from the transmitter to the point at which the tip of the obstruction attains its maximum height, $H_{L S M A X}$ and let $H_{L S M A X}$ be the maximum height attained by the tip of the obstruction at location $X_{L S M A X}$ and distance $d_{\text {LSMAX }}$ from the transmitter. Then,

$$
\begin{gathered}
H_{\text {LSMAX }}=\text { Maximum }\left(H_{e b(x)}+H_{e l(x)}+h_{o b(x)}+\right. \\
\left.\left\{\left(\frac{\mathbf{P}_{C(n)}}{\mathbf{1 0 0}}\right) \sqrt{\frac{n\left(\kappa\left(d_{t(x)}\right)\left(d_{r(x)}\right)\right)}{\left(d_{t(x)}+d_{r(x)}\right)}}\right\}\right)
\end{gathered}
$$

for $\mathrm{x}=X_{L S M A X}$

\subsection{Determination of the Minimum Antenna Height When the Path Inclination Is Zero, $\left(\mathrm{H}_{t}=\mathrm{H}_{r}\right)$}

Let $H_{t}$ be the overall height (in meters) of the transmitter antenna, including the elevation measured from the sea level and the earth bulge at the transmitter. Also, let $H_{r}$ be the overall height (in meters) of the receiver antenna, including the elevation measured from the sea level and the earth bulge at the receiver. Let path inclination be denoted as $\varepsilon_{p}$ where;

$$
\varepsilon_{p}=\frac{\left|H_{t}-H_{r}\right|}{d}
$$


When the path inclination is zero, then,

$$
H_{r}=H_{t}
$$

The transmitter and receiver antenna mast heights are

selected so that the resultant overall heights of the antennas, namely, $H_{t}$ and $H_{r}$ will ensure clear line of sight between the transmitter and the receiver antennas.

$$
H_{t} \geq H_{L S M A X} \text { for all } \mathrm{x} \geq 0
$$

Let $H_{t}=\mathbf{H}_{\mathbf{L S R}(\mathbf{x})}=H_{\text {LSMAX }}$ and let $h_{L S C(x)}$ be the actual LOS clearance height (in meters) from the tip of the obstruction at location $\mathrm{x}$ to the line of sight is given as;

$$
h_{L S C(x)}=H_{o b(x)}-H_{L S M A X}
$$

Note: $h_{L S C(x)}$ is negative if $H_{o b(x)}<H_{L S M A X}$, that is, if the obstruction tip is below the line of sight. In this case, $\mathbf{P}_{\boldsymbol{C}(\boldsymbol{n})}>0$.

$h_{L S C(x)}=0$ if $H_{O b(x)}=H_{L S M A X}$, that is, if the obstruction tip is just on the line of sight. In this case, $\mathbf{P}_{\boldsymbol{C}(\boldsymbol{n})}=0$.

$h_{L S C(x)}$ is positive if $h_{L S C(x)}>H_{L S M A X}$ that is, if the obstruction tip is above the line of sight. In this case, $\mathbf{P}_{\boldsymbol{C}(\boldsymbol{n})}<$ 0

Let $h_{t \text { (mast) }}$ be the height (in meters) of the transmitter antenna mast measured from the ground and let $h_{r \text { (mast) }}$ be the height (in meters) of the receiver antenna mast measured from the ground

Then,

$$
\begin{gathered}
H_{t}=\mathrm{h}_{\mathrm{t}(\text { mast })}+H_{e l t}+H_{e b t} \\
\mathrm{~h}_{\mathrm{t}(\text { mast })}=H_{t}-\left(H_{e l t}+H_{e b t}\right) \\
\mathrm{h}_{\mathrm{t}(\text { mast })}=H_{L S M A X}-\left(H_{e l t}+H_{e b t}\right)
\end{gathered}
$$

But $H_{e b t}=0$, then;

$$
\mathrm{h}_{\mathrm{t}(\text { mast })}=H_{L S M A X}-H_{e l t}
$$

Also,

$$
\begin{gathered}
H_{r}=\mathrm{h}_{\mathrm{r}(\text { mast })}+H_{e l r}+H_{e b r} \\
\mathrm{~h}_{\mathrm{r}(\text { mast })}=H_{r}-\left(H_{e l r}+H_{e b r}\right) \\
\mathrm{h}_{\mathrm{r}(\text { mast })}=H_{L S M A X}-\left(H_{e l r}+H_{e b r}\right)
\end{gathered}
$$

But $H_{e b r}=0$, then;

$$
\mathrm{h}_{\mathrm{r}(\text { mast })}=H_{L S M A X}-H_{e l r}
$$

\section{Results and Discussion}

The path profile used is obtained using online Geocontext-Profiler - an online topographic software available at http://www.geocontext.org/publ/2010/04/profiler/en/. The path profile data are taken for a transmitter located at longitude 7.711747 and latitude 5.178536 and the receiver located at longitude 8.039903and latitude 5.055223. The elevation profile in Table 1 is plotted in figure 2. In Table 1 and figure 2 , there is a total of 512 elevation data points and the link is $40,000 \mathrm{~km}$ long. The link's maximum elevation of 98.3 meters occurred at 11,415.1 meters from the transmitter and the link's minimum elevation of 10.2 meters occurred at a distance of $33,788.8$ meters from the transmitter.

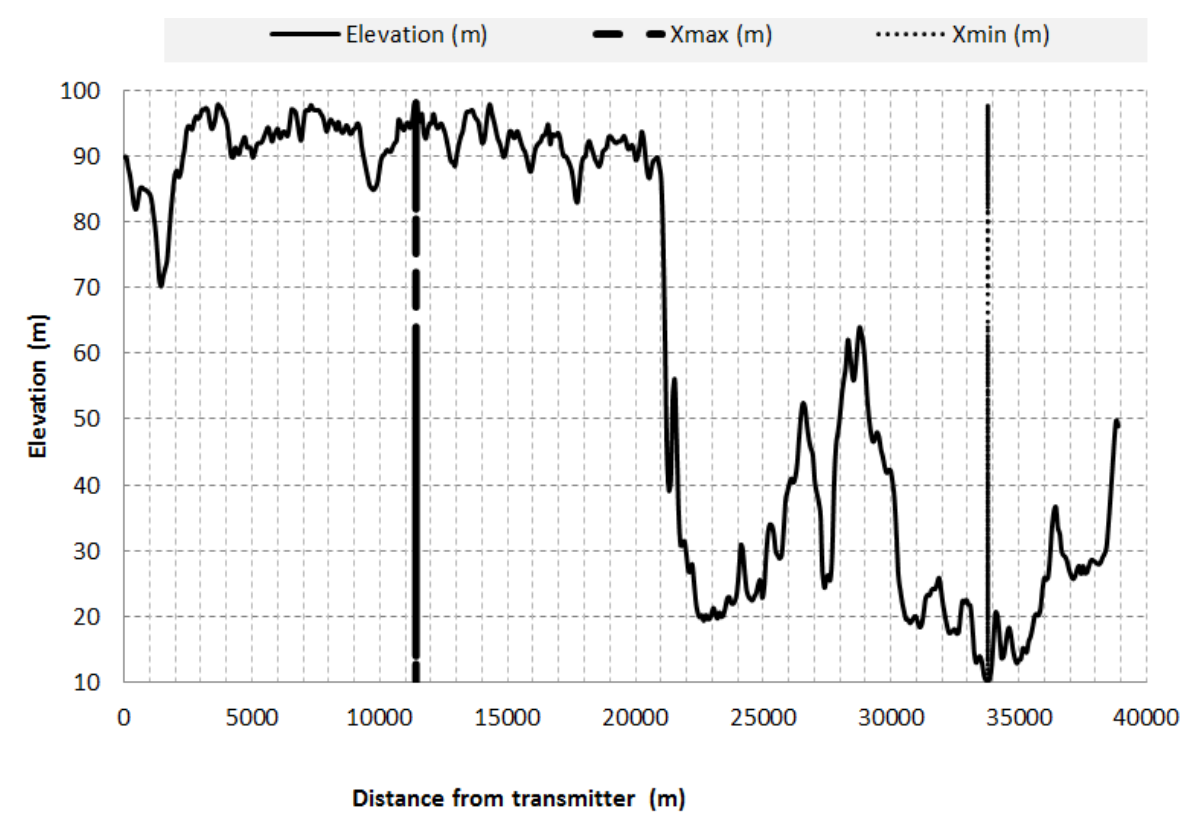

Figure 2. The Elevation profile for the Link. 
Wali Samuel et al: $\quad$ Mathematical Model and Algorithm for Determination of Minimum Antenna Mast

Height for Terrestrial Line of Sight Microwave Link with Zero Path Inclination

Table 1. The Elevation Profile Of The Link.

\begin{tabular}{|c|c|c|c|c|c|}
\hline Elevation Data point & Distance (m) & Elevation (m) & Elevation Data point & Distance (m) & Elevation (m) \\
\hline 1 & 0 & 89.8 & 264 & 20014.6 & 89.4 \\
\hline 12 & 837.1 & 84.9 & 276 & 20927.8 & 88.6 \\
\hline 24 & 1750.3 & 78 & 288 & 21841 & 30.8 \\
\hline 36 & 2663.5 & 94.1 & 300 & 22754.2 & 20.2 \\
\hline 48 & 3576.7 & 96 & 312 & 23667.4 & 23 \\
\hline 60 & 4490 & 90.3 & 324 & 24580.6 & 22.5 \\
\hline 72 & 5403.2 & 92.3 & 336 & 25493.8 & 29.8 \\
\hline 84 & 6316.4 & 93.6 & 348 & 26407 & 47.2 \\
\hline 96 & 7229.6 & 97 & 360 & 27320.2 & 26.8 \\
\hline 108 & 8142.8 & 95.2 & 372 & 28233.5 & 58 \\
\hline 120 & 9056 & 94.4 & 384 & 29146.7 & 49.9 \\
\hline 132 & 9969.2 & 87.6 & 396 & 30059.9 & 40.5 \\
\hline 144 & 10882.4 & 94.2 & 408 & 30973.1 & 20 \\
\hline 151 & 11415.1 & 98.3 & 420 & 31886.3 & 25.9 \\
\hline 156 & 11795.6 & 92.7 & 432 & 32799.5 & 22.4 \\
\hline 168 & 12708.9 & 90.5 & 444 & 33712.7 & 10.3 \\
\hline 180 & 13622.1 & 97 & 445 & 33788.8 & 10.2 \\
\hline 192 & 14535.3 & 94 & 456 & 34625.9 & 18.3 \\
\hline 204 & 15448.5 & 93 & 468 & 35539.1 & 18.3 \\
\hline 216 & 16361.7 & 93 & 480 & 36452.4 & 36.7 \\
\hline 228 & 17274.9 & 90 & 492 & 37365.6 & 27.7 \\
\hline 240 & 18188.1 & 92.3 & 504 & 38278.8 & 29 \\
\hline 252 & 19101.3 & 92.6 & 512 & 38887.6 & 48.9 \\
\hline Maximum Elevation & Maximum & Maximum Elevation Distance & Minimum Elevation & minimum & Minimum Elevation Distance \\
\hline Data point & Elevation (m) & From The Transmitter, Xmax (m) & Data point & Elevation (m) & From The Transmitter, Xmin (m) \\
\hline 151 & 98.3 & 11415.1 & 445 & 10.2 & 33788.8 \\
\hline
\end{tabular}

Table 2. The Link Clearance Parameters.

\begin{tabular}{|c|c|c|c|c|c|c|c|}
\hline $\begin{array}{l}\text { Elevation Data } \\
\text { Point Number } \\
\end{array}$ & $\begin{array}{l}\text { Distance } \\
(\mathrm{m})\end{array}$ & $\begin{array}{l}\text { Elevation } \\
(\mathrm{m})\end{array}$ & $\begin{array}{l}\text { Earth Bulge } \\
(\mathrm{m})\end{array}$ & $\begin{array}{l}\text { Obstruction } \\
\text { height(m) }\end{array}$ & $\begin{array}{l}\text { Radius Of Fresnel } \\
\text { Zone } 1(\mathrm{~m})\end{array}$ & $\begin{array}{l}\text { Line Of Sight } \\
\text { Clearance Height (m) }\end{array}$ & $\begin{array}{l}\text { Percentage Clearance Of } \\
\text { Fresnel Zone } 1(\%)\end{array}$ \\
\hline 1 & 0 & 89.8 & 0 & 99.8 & 0 & -58.8185 & 0 \\
\hline 24 & 1750.32 & 81.8 & 3.8 & 91.8 & 12.93 & -66.8461 & -517 \\
\hline 72 & 5403.17 & 103 & 10.6 & 113 & 21.57 & -45.6927 & -212 \\
\hline 96 & 7229.59 & 110.5 & 13.5 & 120.5 & 24.26 & -38.1905 & -157 \\
\hline 120 & 9056.01 & 110.3 & 15.9 & 120.3 & 26.36 & -38.4033 & -146 \\
\hline 168 & 12708.86 & 110.1 & 19.6 & 120.1 & 29.25 & -38.5907 & -132 \\
\hline 189 & 14306.98 & 118.6 & 20.7 & 128.6 & 30.07 & -30.0722 & -100 \\
\hline 192 & 14535.28 & 114.8 & 20.8 & 124.8 & 30.17 & -33.8383 & -112 \\
\hline 216 & 16361.71 & 114.7 & 21.7 & 124.7 & 30.79 & -33.9353 & -110 \\
\hline 256 & 19405.74 & 114.5 & 22.2 & 124.5 & 31.18 & -34.1746 & -110 \\
\hline 264 & 20014.55 & 111.6 & 22.2 & 121.6 & 31.17 & -37.0346 & -119 \\
\hline 288 & 21840.97 & 52.7 & 21.9 & 62.7 & 30.94 & -95.9249 & -310 \\
\hline 312 & 23667.4 & 44.2 & 21.2 & 54.2 & 30.44 & -104.497 & -343 \\
\hline 432 & 32799.51 & 34.1 & 11.7 & 44.1 & 22.66 & -114.522 & -505 \\
\hline 456 & 34625.93 & 27 & 8.7 & 37 & 19.48 & -121.665 & -625 \\
\hline 480 & 36452.36 & 41.9 & 5.2 & 51.9 & 15.11 & -106.78 & -707 \\
\hline 504 & 38278.78 & 30.3 & 1.4 & 40.3 & 7.74 & -118.322 & -1528 \\
\hline 512 & 40000.00 & 48.9 & 0 & 58.9 & 0 & -99.7385 & - \\
\hline
\end{tabular}

Table 3. The Antenna Mast Heights and Other Link Clearance Parameters.

\begin{tabular}{lll}
\hline Link Parameter & Value & \\
\hline Height of Transmitter Antenna Mast, (m) & 68.8 \\
Height of Receiver Antenna Mast, (m) & 109.7 & 158.7 \\
Height of Line Of Sight (m) = Transmitter Antenna Height (m) = Receiver Antenna Height (m) & 128.58 \\
Maximum Height Of The Tip Of Obstruction (m) & 14306.98 \\
Distance of Maximum Obstruction Height, (m) & -30.07 \\
Minimum Line Of Sight Clearance Height, & 14306.98 \\
Distance of Minimum Line Of Sight Clearance Height From The Transmitter, (m) & 30.07 \\
Radius Of The First Fresnel Zone At The Minimum Clearance Height (m) & -100 \\
Percentage Clearance of First Fresnel Zone At The Minimum Clearance Height (\%) & -100 \\
Specified Percentage Clearance (\%) & \\
\hline
\end{tabular}


In Table 2 the maximum obstruction height of $128.58 \mathrm{~m}$ occurred at a distance of $14306.98 \mathrm{~m}$ from the transmitter. The Percentage Clearance of $100 \%$ is achieved at the maximum obstruction point. This is equal to the $100 \%$ clearance given in the link design specification. In Table 3, the transmitter and receiver antenna are at the same line of sight height of $158.7 \mathrm{~m}$. However, the transmitter antenna mast height is $68.8 \mathrm{~m}$ while the receiver antenna mast height is $109.7 \mathrm{~m}$. Maximum earth bulge of 22.2 occurred at the middle of the like at a distance of $19405.74 \mathrm{~m}$ from the transmitter.

\section{Conclusion}

Mathematical model and algorithm for determination of minimum antenna mast height for terrestrial line of sight microwave link with zero path inclination is presented. The mathematical expressions developed are used for computing relevant link parameters while the algorithm gives the procedure for using the mathematical expressions for computing the minimum antenna mast heights. Sample $\mathrm{Ku}$-band microwave link is used to demonstrate the application of the model and algorithm. The result shows that the model can be used to ensure that the specified percentage clearance is achieved through the application of the models presented in this paper.

\section{References}

[1] Al Mahmud, M. R. (2009). Analysis and planning microwave link to established efficient wireless communications (Doctoral dissertation, Blekinge Institute of Technology).

[2] McKown, J. W., \& Hamilton, R. L. (1991). Ray tracing as a design tool for radio networks. IEEE Network, 5(6), 27-30.

[3] Adler, R. B., Chu, L. J., \& Fano, R. M. (1960). Electromagnetic energy transmission and radiation.

[4] Feuerstein, M. J., Blackard, K. L., Rappaport, T. S., Seidel, S. Y., \& Xia, H. H. (1994). Path loss, delay spread, and outage models as functions of antenna height for microcellular system design. IEEE Transactions on Vehicular Technology, 43(3), 487-498.

[5] Angulo, I., De La Vega, D., Cascón, I., Cañizo, J., Wu, Y., Guerra, D., \& Angueira, P. (2014). Impact analysis of wind farms on telecommunication services. Renewable and Sustainable Energy Reviews, 32, 84-99.

[6] Wibling, O. (1998). Terrain Analysis with Radio Link
Calculations for a Map Presentation Program. Terrain, 98, 12-08.

[7] Adediji, A. T., Mandeep, J. S., \& Ismail, M. (2014). Meteorological Characterization of Effective Earth Radius Factor (k-Factor) for Wireless Radio Link Over Akure, Nigeria. Mapan, 29 (2), 131-141.

[8] Serdega, V., \& Ivanovs, G. (2015). Refraction seasonal variation and that influence on to $\mathrm{GHz}$ range microwaves availability. Elektronika ir Elektrotechnika, 78 (6), 39-42.

[9] Mufti, N., Ullah, H., Rehman, S., \& Din, I. (2015, January). Analysis of tropospheric radio refractive conditions in Karachi, Pakistan. In 2015 12th International Bhurban Conference on Applied Sciences and Technology (IBCAST) (pp. 617-619). IEEE.

[10] Nyete, A. M., \& Afullo, T. J. O. (2013). Seasonal distribution modeling and mapping of the effective earth radius factor for microwave link design in South Africa. Progress In Electromagnetics Research B, 51, 1-32.

[11] Zaidi, K. S., Jeoti, V., Iqbal, A., \& Awang, A. (2014, June). Feasibility of trans-horizon, high-capacity maritime wireless backhaul communication link. In Intelligent and Advanced Systems (ICIAS), 2014 5th International Conference on (pp. 1-6). IEEE.

[12] Dalbakk, L. E. (2014). Antenna System for Tracking of Unmanned Aerial Vehicle.

[13] Mazar, H. (1991, March). LOS radio links, clearance above tall buildings. InElectrical and Electronics Engineers in Israel, 1991. Proceedings., 17th Convention of (pp. 145-148). IEEE.

[14] Dalbakk, L. E. (2014). Antenna System for Tracking of Unmanned Aerial Vehicle.

[15] Arzubi, A. A., Castro Lechtaler, A., Foti, A., Fusario, R., García Garino, C., \& García Guibout, J. (2010). Design of a Trans-Horizon radio link for ultra high and super high frequencies. In XVI Congreso Argentino de Ciencias de la Computación.

[16] Haykin, S. S., Moher, M., \& Koilpillai, D. (2011). Modern wireless communications. Pearson Education India.

[17] Kraus, H. G. (1989). Huygens-Fresnel-Kirchhoff wave-front diffraction formulation: spherical waves. JOSA A, 6 (8), 1196-1205.

[18] Beyer, J. (2004). An approximate approach to predict multiple screen diffraction in the case of grazing incidence. Radio science, 39 (4).

[19] Capon, J. (1976). Multipath parameter computations for the MLS simulation computer program (No. ATC-68). Lincoln Laboratory, Massachusetts Institute of Technology. 